\title{
The clinical-phenotype continuum in DYNC1H1-related disorders- genomic profiling and proposal for a novel classification
}

\author{
Lena-Luise Becker $\mathbb{1}^{1} \cdot$ Hormos Salimi Dafsari $^{2}$ - Jens Schallner ${ }^{3}$. Dalia Abdin ${ }^{4,5}$ - Michael Seifert ${ }^{6}$. \\ Florence Petit $\mathbb{D}^{7,8} \cdot{\text { Thomas Smol } \mathbb{C}^{7,9} \cdot \text { Levinus } \text { Bok }^{10} \cdot \text { Lance Rodan }^{11} \cdot \text { Ingrid Krapels }}^{12} \cdot$ Stephanie Spranger $^{13}$. \\ Bernhard Weschke $\mathbb{I D}^{1} \cdot$ Katherine Johnson $^{14} \cdot$ Volker Straub $\left.^{10}\right)^{14} \cdot$ Angela M. Kaindl $^{1} \cdot$ Nataliya Di Donato $^{15}{ }^{15}$. \\ Maja von der Hagen ${ }^{3} \cdot$ Sebahattin Cirak $^{16}$
}

Received: 25 February 2020 / Revised: 18 June 2020 / Accepted: 19 June 2020 / Published online: 12 August 2020

(c) The Author(s) 2020. This article is published with open access

\begin{abstract}
Mutations in the cytoplasmic dynein 1 heavy chain gene $(D Y N C 1 H 1)$ have been identified in rare neuromuscular (NMD) and neurodevelopmental (NDD) disorders such as spinal muscular atrophy with lower extremity dominance (SMALED) and autosomal dominant mental retardation syndrome 13 (MRD13). Phenotypes and genotypes of ten pediatric patients with pathogenic $D Y N C 1 H 1$ variants were analyzed in a multi-center study. Data mining of large-scale genomic variant databases was used to investigate domain-specific vulnerability and conservation of DYNC1H1. We identified ten patients with nine novel mutations in the DYNCIHI gene. These patients exhibit a broad spectrum of clinical findings, suggesting an overlapping disease manifestation with intermixed phenotypes ranging from neuropathy (peripheral nervous system, PNS) to severe intellectual disability (central nervous system, CNS). Genomic profiling of healthy and patient variant datasets underlines the domain-specific effects of genetic variation in $D Y N C 1 H 1$, specifically on toleration towards missense variants in the linker domain. A retrospective analysis of all published mutations revealed domain-specific genotype-phenotype correlations, i.e., mutations in the dimerization domain with reductions in lower limb strength in DYNC1H1-NMD and motor domain with cerebral malformations in DYNC1H1-NDD. We highlight that the current classification into distinct disease entities does not sufficiently reflect the clinical disease manifestation that clinicians face in the diagnostic work-up of DYNC1H1-related disorders. We propose a novel clinical classification for $D Y N C 1 H 1$-related disorders encompassing a spectrum from DYNC1H1-NMD with an exclusive PNS phenotype to DYNC1H1-NDD with concomitant CNS involvement.
\end{abstract}

\section{Introduction}

Mutations in the cytoplasmic dynein 1 heavy chain 1 gene (DYNC1H1) were first described in 2010 in autosomal

These authors contributed equally: Lena-Luise Becker, Hormos Salimi Dafsari

Supplementary information The online version of this article (https:// doi.org/10.1038/s10038-020-0803-1) contains supplementary material, which is available to authorized users.

Maja von der Hagen

Maja.Hagenv.der@uniklinikum-dresden.de

Sebahattin Cirak

sebahattin.cirak@uk-koeln.de

Extended author information available on the last page of the article dominant lower extremity-predominant spinal muscular atrophy 1 (SMALED1; MIM\#158600) [1, 2]. Subsequent reports linked $\mathrm{DYNClH1}$ mutations to neuromuscular (NMD, e.g., axonal Charcot-Marie-Tooth disease type 20 (CMT20; MIM\#614228) [3, 4]) and neurodevelopmental disorders (NDD), e.g., malformations of cortical development (MCD), autosomal dominant mental retardation 13 (MRD13; MIM\#614563) [5], and hereditary spastic paraplegia. Approximately 120 patients with $D Y N C 1 H 1$ mutations have been published so far.

DYNClHI encodes for a subunit of the cytoplasmic dynein complex. $\mathrm{DYNClHI}$ comprises four major protein regions, i.e., tail domains (amino acid residues 1-1373 and 4222-4646), linker domain (aa 1374-1867), motor domains with AAA domains (ATPases associated with a variety of cellular activities, aa 1868-3168 and 3553-4221), and the stalk or microtubule-binding domain (MTBD, aa 
3169-3552) (Fig. 1b). Recent molecular biological reports have shed light on the functional role of $\mathrm{DYNClHI}$ in neuronal development. The dynein-dynactin complex is, together with cargo adapters (e.g., BICD2) [6-8], key for trafficking of cellular cargo to the minus-end on microtubules, for spindle pole organization, Golgi maintenance,
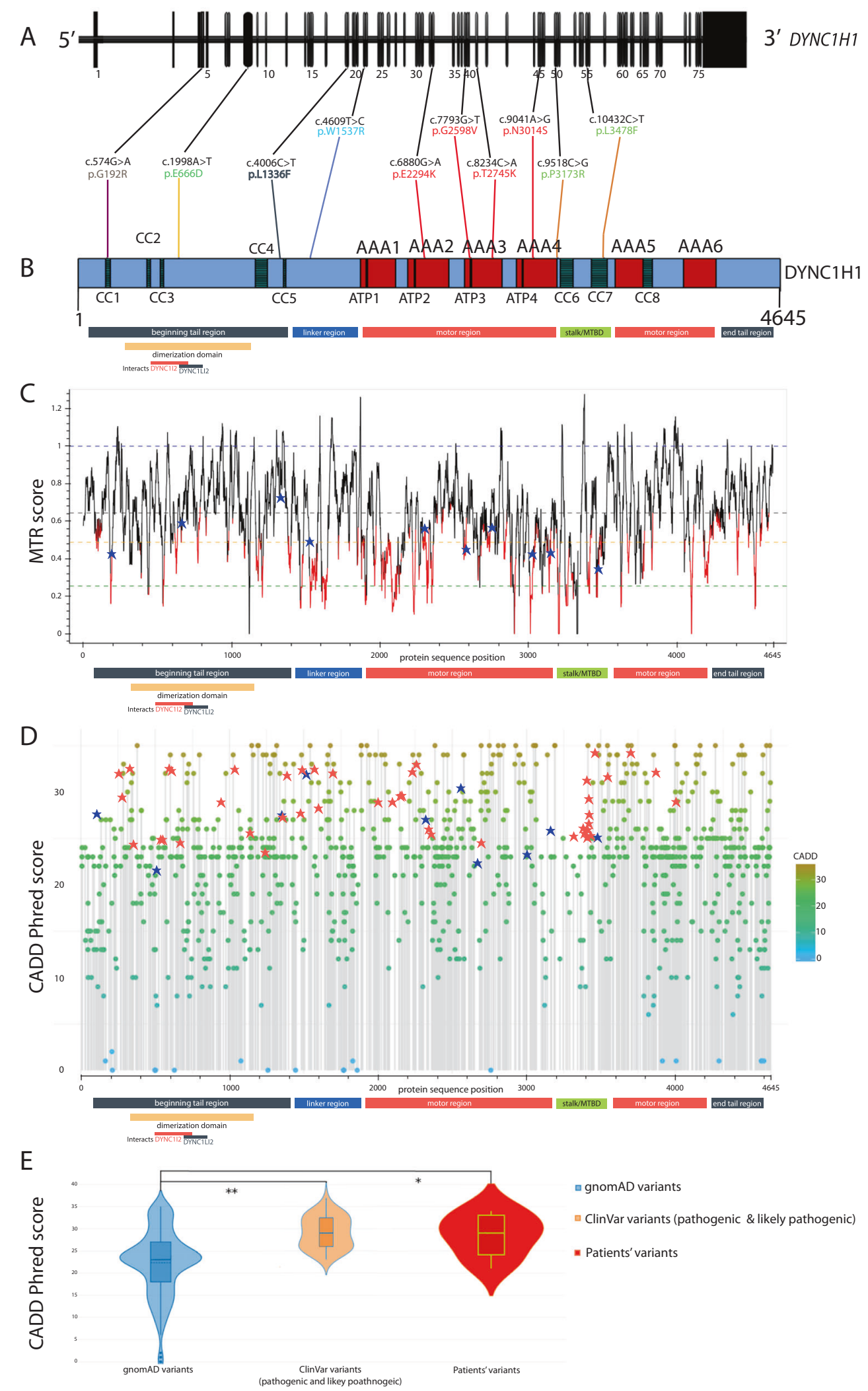
Fig. 1 Overview of DYNC1H1 variants identified in this study. Calculated MTR and CADD-Phred score values for the variants from the healthy population and our patient collective show that pathogenic DYNC1H1 mutations cluster in regions of less genetic heterogeneity, specifically in highly conserved domains. a Ten variants in the DYNC1H1 gene (NM_001376.5, 78 exons) identified in our patients and concomitant position in b. DYNC1H1 protein structure (Q14204); pictogram with protein domains: coiled coil domain (CC, gray), ATPase associated with various cellular activities domain (AAA, red), ATP-binding region in AAA domain (ATP, dark brown), rest of protein in blue. We noted all regions (beginning tail region gray, linker region dark blue, motor region red, stalk/microtubule-binding domain green, end tail region gray) and specified the dimerization domain in yellow with interaction partners DYNC1I2 and DYNC1LI2 noted below. The mutations on protein level are presented in the above-mentioned color scheme. c Missense tolerant ratio (MTR) gene viewer result for DYNC1H1 (ENST00000360184) with window size 21 (http://biosig.unimelb.edu.au/mtr-viewer/); patients' variants are marked with blue crosses. Protein regions noted below as in $\mathbf{b}$. d CADD-Phred scores of all gnomAD variants with ClinVar patient variants (marked with red asterisks) and our patient's variants (marked with blue asterisks), score $>20$ indicates likely pathogenic computation, score $>30$ indicates pathogenic [48]. In general, CADD is a genelevel scoring for potential proxy-deleterious variants and has to be treated with caution. The linker mutations in our patient collective show amino acid exchanges with more significant changes in physicochemical properties when compared with variants from a healthy population dataset. The patients' mutations in the motor region are found in highly conserved AAA domains with higher CADD-Phred score values. However, the pathogenic mutations from patients are in regions where allele frequencies and high CADD-Phred scores are "thinned out". For the raw data, please see Supplementary Table 2. Protein regions noted below as in b. e Violin plot for CADDPhred scores for variants recorded in gnomAD database (left in blue, https://gnomad.broadinstitute.org/); likely pathogenic and pathogenic variants according to ClinVar (middle in orange, https://www.ncbi. nlm.nih.gov/clinvar/), and ten patients variants (right in red), please see Supplementary Table 2 for raw data. Variance analysis (ANOVA, SigmaPlot 12.5, SYSTAT, USA) revealed significant differences between the groups "gnomAD variants" and "ClinVar variants" $(* * p$ $<0.01)$ as well as the groups "gnomAD variants" and "patients' variants" in our ten patients $\left({ }^{*} p<0.05\right)$. There was no significant difference between the groups "ClinVar variants" and "patients' variants"

endolysosomal processing, and nuclear positioning migration during mitosis in eukaryotic cells $[6,9,10]$.

In this multi-center study, we report the clinical course of

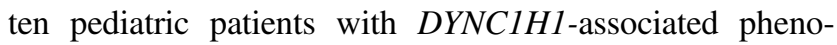
types with nine novel pathogenic variants, highlighting the broad clinical heterogeneity of dyneinopathies and proposing a new clinical classification for $\mathrm{DYNC} 1 \mathrm{H} 1$-related disorders.

\section{Materials and methods}

\section{Genetic investigations}

We included ten patients (P1-P10) with DYNC1H1 (NM_001376.5) mutations in this multi-center study.
All parents and/or patients gave informed consent. The study was approved by the ethics committee of the Medical Faculty, University of Cologne (17-096).

Genetic investigations of P1 via a next-generation sequencing panel (MYO-SEQ project in Newcastle University) revealed a heterozygous variant in $\mathrm{DYNClH1}$ after various genes had been sequenced without detection of putative pathogenic variants at the time when whole-exome sequencing (WES) was not easily available [11]. Subsequently, the DYNC1H1 variant was identified by Sanger Sequencing in the father. Later, P1 and both parents underwent trio-WES and the DYNC1H1 variant was confirmed in P1 and his father [11].

P2 was diagnosed via a targeted next-sequencing gene panel following enrichment using highly specific Molecular Inversion Probes [12]. For P3, we performed a commercial targeted next-generation sequencing panel including coding regions of 61 NMD genes (spinal muscular atrophy and limb-girdle muscular dystrophies panel, MGZ München).

P4 received trio-WES after enrichment for the IDT $\mathrm{xGene}$ exome research panel followed by $2 \times 150 \mathrm{bp}$ sequencing with a mean target coverage of 100 -fold on Illumina NextSeq500 Sequencer. Alignment (mapping to GRCh37/hg19), variant identification (SNPs and indels), variant annotation, and filtering were performed using the CLC Biomedical Genomics Workbench (Qiagen, Hilden, Germany). Variants were filtered with a focus on proteinaltering variants (missense, frame-shift, splice-site, and premature stop-codons) rare or absent (de novo variants) from public databases (gnomAD and 1000 Genomes project) as previously described [13].

In P5, we performed trio-WES after enrichment with Agilent SureSelect V6 kit (Agilent, USA) on an Illumina HiSeq 4000 Sequencer (Illumina, USA) with $2 \times 75$ bp sequencing protocol according to the manufacturer's bestpractice protocol, a mean coverage of 85 for the patient and father and a mean coverage of 80 for the mother was achieved $[14,15]$. The sequencing data were analyzed using a version of the Cologne Center for Genomics exome pipeline, version 2.20 [16]. The annotated variant lists were uploaded to the Cologne Center for Genomics Varbank (https://varbank.ccg.uni-koeln.de) database for variant filtering. Since a trio-WES was available, we additionally performed calling and filtering for de novo variants using deNovoGear [17].

For P6, the $\mathrm{DYNC1H1}$ variant in patient and parents was found by a commercial next-generation sequencing gene panel for fetal akinesia (ID 078.03, MGZ München).

In P7, we performed WES after enrichment with the NimbleGen MedExome kit (Roche NimbleGen, Basel, Switzerland) on an Illumina HiSeq 4000 Sequencing System (Illumina, San Diego, CA, USA) with $2 \times 150 \mathrm{bp}$ sequencing protocol according to the manufacturer's best- 
practice protocol. The variant calling and filtering pipeline were described earlier elsewhere [14].

In P8, trio-WES was performed after enrichment using the SOLiD-optimized SureSelect All Human Exon Kit (50 Mb; Agilent, Technologies, Santa Clara, CA, USA), followed by sequencing on 5500XL sequencers (Life Technologies, Carlsbad, CA, USA). Quality control parameters were checked throughout the laboratory workflow. Sequence reads were aligned to the human genome (hg19) using Lifescope v2.1 (Life Technologies), followed by variant calling on the aligned sequence. Variants were annotated using a custom analysis pipeline. Samples were automatically checked for quality (e.g., median coverage). For further information on the sequencing procedure, please see previously published data [18].

In P9, we performed WES with Agilent Clinical Research Exome Kit on an Illumina HiSeq 2000 with $2 \times$ $100 \mathrm{bp}$ reads, mean depth of coverage of $103 \times$, and quality threshold of $95.9 \%$ (percentage of XomeDx which is covered by at least ten sequence reads/ $10 \times$ coverage). The data were aligned to reference NM sequence based on GRCh37/ hg19 and analyzed for sequence variants using a customdeveloped analysis tool (Xome Analyzer).

In P10, we performed a next-generation sequencing panel targeting PAFAH1B1, KIF5C, KIF2A, TUBG1, CRADD, and $D Y N C 1 H 1$, after using enrichment with the Agilent insolution hybridization technology followed by sequencing on an Illumina HiSeq Sequencing system (Illumina, USA). The analysis included a next-generation sequencing-based copy number variant calling and analysis. The panel was supplemented with an MLPA-based deletion- and duplication analyses for PAFAHIBI.

We performed dideoxy seqeucning for confirmation of all the patients' DYNC1H1 variants and for further cosegregation analyses, except for P1 and P10.

All variants were scored based on the classification by the standards and guidelines of the American College of Medical Genetics and Genomics-American College of Molecular Pathology (ACMG) for the interpretation of variants [19].

\section{Genotype-phenotype analyses including retrospective literature analysis}

To further characterize the phenotype of patients with mutations in $D Y N C 1 H 1$, we searched via "Pubmed" for publications of patients with mutations in $\mathrm{DYNClHl}$ from 01.01.2010 to 01.01.2020 and identified 24 publications with 120 patients $[1,3-5,20-38]$. We used IBM SPSS Statistics 25 (IBM Corp. Released 2017, Armonk, NY) to generate a database of all patients including our ten patients $(n=130)$ and assigned each patient for 12 clinical manifestations (upper and lower limb strength, intellectual disability (ID), behavior, seizures, MRI abnormalities including pachygyria, heterotopias, enlarged ventricles, hypoplasia of corpus callosum, of the brain stem, and the cerebellum) either a physiological phenotype " 0 " or an abnormal phenotype " 1 ". Where information was not available or not specifically mentioned in the publications, we did not assign a number and marked it as missing. For an overview of all patients included in the analyses, please see Supplementary Table 1.

\section{Statistical analyses}

We performed further in silico and genomic analysis [39] for all variants, checking in population databases such as gnomAD (http://gnomad.broadinstitute.org), 1000 Genomes [40], GME [41], and ExAC browser (http://exac.broa dinstitute.org/). To evaluate the pathologic potential of the resulting variants, we used MutationTaster (http://www. mutationtaster.org/), SIFT (http://sift.jcvi.org/), PolyPhen2 (http://genetics.bwh.harvard.edu/pph2/), GERP ++ [42], REVEL [43], CADD-Phred (https://cadd.gs.washington. edu/), and MutPred2 (http://mutpred.mutdb.org/).

The missense tolerance ratio (MTR, Fig. 1c) calculates the number of observed missense DNA variations relative to the number of all observed (missense and synonymous) single base variants, then divided by the number of expected missense mutations relative to the number of all possible variations in that segment [44]. To evaluate the evolutionary conservation, we performed multiple sequence alignment for all patients using Clustal Omega (https://www.ebi.ac.uk/ Tools/msa/clustalo/) (Fig. 2).

In order to judge the pathogenicity on a domain-specific protein level and investigate the domain-specific vulnerability and conservation of $D Y N C 1 H 1$, we analyzed datasets on $\mathrm{DYNC1H1}$ variants in a healthy population (gnomAD database) with a patient collective. We performed twofactor analysis of variances (ANOVA, SigmaPlot 12.5, SYSTAT, USA) for CADD-Phred score values and MTR score values between the groups "reports" (healthy subjects vs patients) and the groups "protein regions" (tail vs linker vs motor vs MTBD). For these analyses, we pooled the ten patients from our collective with pathogenic and likely pathogenic $\mathrm{DYNClH1}$ mutation reports from the ClinVar database (Supplementary Table 2). For all statistical analyses, we performed one-factor or two-factor ANOVA as specified in the text, and we report significant differences with a $p$ value below 0.05 or lower.

For statistical analyses of the genotype-phenotype analyses, we performed Pearson's Chi-Square test, Lambda, Phi, and Cramer V test (Supplementary Table 3) to correlate the phenotype to the localization of the mutations categorized by the domain (beginning tail, dimerization, linker, motor domain). We plotted the results with a balloon plot 
A

C. elegans TVQKCETEAEAALLHLQQNIDIP D. melanogaster SVEKKLAELEMGLLHLQQNIDIP Danio rerio SVEKKIAELEMGLLHLQQNIEIP Mus musculus SVEKKIAELEMMGLLHLQONTEIP Homo sapiens SVEKKIAELEMGLLHLOONIEIP P1, p.G192R SVEKKIAELEMRLLHLQQNIEIP $\begin{array}{ll}\text { C. elegans } & \text { ELAAMKDVWKALQPVYTGIDEAKE } \\ \text { D. melanogaster } & \text { ELQDLRGVWSELSKVWTQIDETRE } \\ \text { Danio rerio } & \text { ELQDLKGVWSELSKVWEQIDQMK- } \\ \text { Gallus gallus } & \text { ELQDLKGVWSELSKVWEQIDQMK- } \\ \text { Mus musculus } & \text { ELQDLKGVWSELSKVWEQIDQMK- } \\ \text { Homo sapiens } & \text { ELQDLKGVWSELSKVWEQIDQMK- } \\ \text { P3, p.L1336F } & \text { ELQDLKGVWSEFSKVWEQIDQMK- }\end{array}$

E C. elegans GFLERLNTLLANGEVPGLFEGDE Delanogaster GFLERMNTLLANGEVPGLFEGDE Gallus gallus GFLERMNTLLANGEVPGLFEGDE Mus musculus GFLERMNTLLANGEVPGLFEGDE Homo sapiens GFLERMNTLLANGEVPGLFEGDE P5, p.N3014S GFLERMNTLLASGEVPGLEEGDE $* * * * *, * * * * * * * * * * * * * * * * *$

C. elegans VYVDYPGQTSLQQIYGTFNRAML D. melanogaster IYVDYPGETSLKQIYGTFSRAMI Danio rerio VYVDYPGPASLTOIYGTFNRAMI Gallus gallus VYVDYPGPASLTQIYGTFNRAML Mus musculus VYVDYPGPASLTQIYGTFNRAMI Homo sapiens VYVDYPGPASLTOIYGTFNRAMI P7. p.T2745K VYVDYPGPASLKQIYGTFNRAMI $: * * * * * *: * * * * * * * * . * * * *$

C. elegans EHKPLVLCGPPGSGKTMTLLAAI D. melanogaster EHKPLVLCGPPGSGKTMTLFSAL Danio rerio EHKPLVLCGPPGSGKTMTLFSAL Gallus gallus EHKPLVLCGPPGSGKTMTLFSAL Mus musculus EHKPLVLCGPPGSGKTMTLFSAL Homo sapiens EHKPLVLCGPPGSGKTMTLFSAL P9, p.G2598V EHKPLVLCGPPVSGKTMTLFSAI $* * * * * * * * * * * * * * * * * * \ldots, \ldots * *$ Gallus gallus SVEKKIAELEMGLLHLQQNIEIP $: *:^{*}::^{*} * * * * * * * * * * *$ Danio rerio GFLERMNTLLANGEVPGLFEGDE
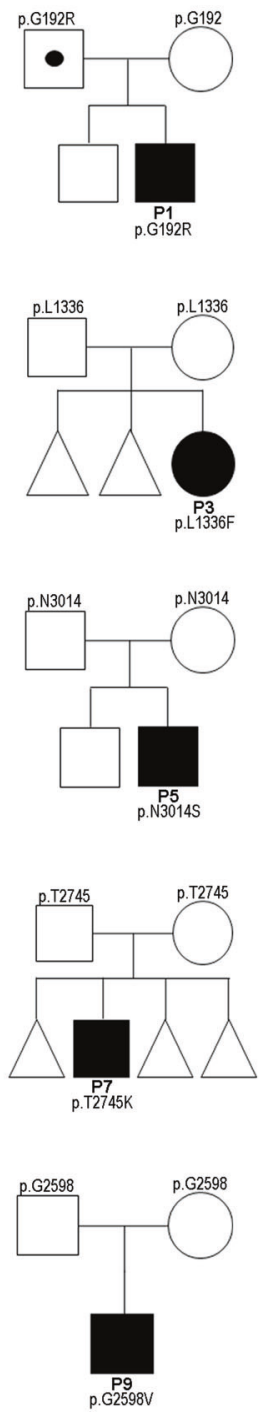

Fig. 2 Pedigrees of P1-10 and multi-species sequence alignment of the mutated proteins. a Pedigree and multi-species sequence alignment of $\mathrm{P} 1$, to note, father is also heterozygous but does not show any symptoms. b-j Multi-species sequence alignment and pedigrees of P2-10. Multi-species sequence alignment was performed using Clustal Omega (Caenorhabditis elegans, NP_491363.1; Drosophila

using R 3.6.3 GUI 1.70 El Capitan build (7735) for MacOS and the packages ggpubr, ggplot2, and magrittr (Fig. 4b, c).

\section{Results}

\section{Clinical findings}

We report ten pediatric patients (P1-P10) with overlapping DYNC1H1-associated phenotypes, onset in infancy, and little to no disease progression (Table 1, Fig. 2). Motor development was delayed in all patients except for P10. Eight out of ten patients had muscular weakness and

\section{C. elegans D. melanogaster Danio rerio Gallus gallus Mus musculus Homo sapiens P4, p. E2294K}

F

C. elegans D. melanogaster Danio rerio Gallus gallus

Mus musculus Homo sapiens P6, p.W1537R

H

C. elegans D. melanogaster Danio rerio

Gallus gallus

Mus musculus

Homo sapiens

P8, pP3173R.

SVQEKVNRSTELLSSLRSERDRW NVQAKVDRSIALIKSLNIERERW AVEAKVNRSTALLKSLSAERERI AVEAKVNRSTALLKSLSAERERW AVEAKVNRSTALLKSLSAERERW AVEAKVNRSTALLKSLSAERERW AVEAKVNRSTAFLKSLSAERERW ${ }^{*}: * *: * * \quad: * . * * \quad * *: * *$

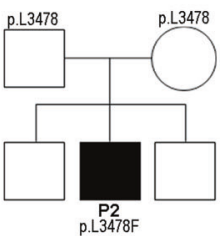

VIRKI IDNVRGFADRROWI TFDG LRKI I DNVRGE INKRQWI IFDG VLRKI IDNVRGE LQKRQWI IFDG VLRKI I DNVRGE LQKRQWI I FDG VLRKI IDNVRGE LQKRQWIVFDG VLRKI IDSVRGE LQKRQWIVFDG VLRKI IDSVRGKLQKRQWIVFDG $\because * * * * * * * * *: \because * * * * * * * *$
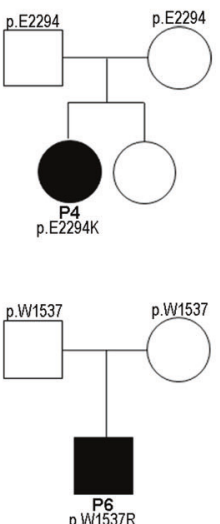

KLNR TMALFDVRIDVQRRWVYLE $* * *: * *: * * * * * * * * * * * * * *$

KLNKINAMFDVWIDVQRRWVYLE KINTNALFVWIDVQRRWVYLE KLNR IMALFDVWIDVQRRWVYLE KLNRIMALFDVWIDVQRRWVYLE KLNRIMALFDVWIDVQRRWVYLE

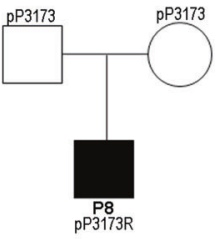

$\mathbf{J}$ $\begin{array}{ll}\text { C. elegans } & \text { ERQLQRYMKRVEDVLGKQWENHV } \\ \text { D. melanogaster } & \text { DNQLTMYLKRVEDVLGKGWETHI } \\ \text { Danio rerio } & \text { DRQLTAYMKRVEDVLGKGWENHV } \\ \text { Gallus gallus } & \text { DRQLTAYMKRVEDVLGKGWENHV } \\ \text { Mus musculus } & \text { DRQLTAYMKRVEDVLGKGWENHV } \\ \text { Homo sapiens } & \text { DRQLTAYMKRVEDVLGKGWENHV } \\ \text { P10, p.E666D } & \text { DRQLTAYMKRVDDVLGKGWENHV } \\ & : . * \star *: * * * * * * * * * * *\end{array}$

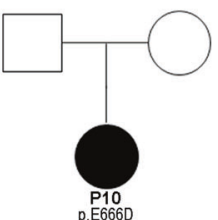

melanogaster, NP_001261430.1; Danio rerio, NP_001036210.1; Gallus gallus, XP_015143281.1; Mus musculus, NP_084514.2; Homo sapiens, NP_001367.2). $\square$ male, not affected; $\bigcirc$, female, not affected; with dot, carrier; $\square$, male, affected; $\boldsymbol{O}$, female, affected; *, fully conserved: conserved between groups of strongly similar properties; conservation between groups of weakly similar properties

atrophy predominantly of the lower limbs (P1-P8) with "crouching" movements and three patients exhibited weakness also of the upper limbs (P1, P6, and P7). Deep tendon reflexes (DTR) were normal in three patients (P4, P9, and P10); all other patients had reduced DTR in the lower limb but normal DTR in the upper limb.

Feet deformities were present in five patients (pes cavus: P1; pes equinus: $\mathrm{P} 2$, P3; pes equinovatus: $\mathrm{P} 4$, pes adductus: P6), contractures in two patients (hips: P2, P6; knees: P2), spine deformities in three patients (lumbar hyperlordosis: P1, P3; merging of dens tip and atlas anterior arch: P6), and one patient displayed with hip dysplasia (P1). Gait was assessed in eight patients and abnormal in all with the 


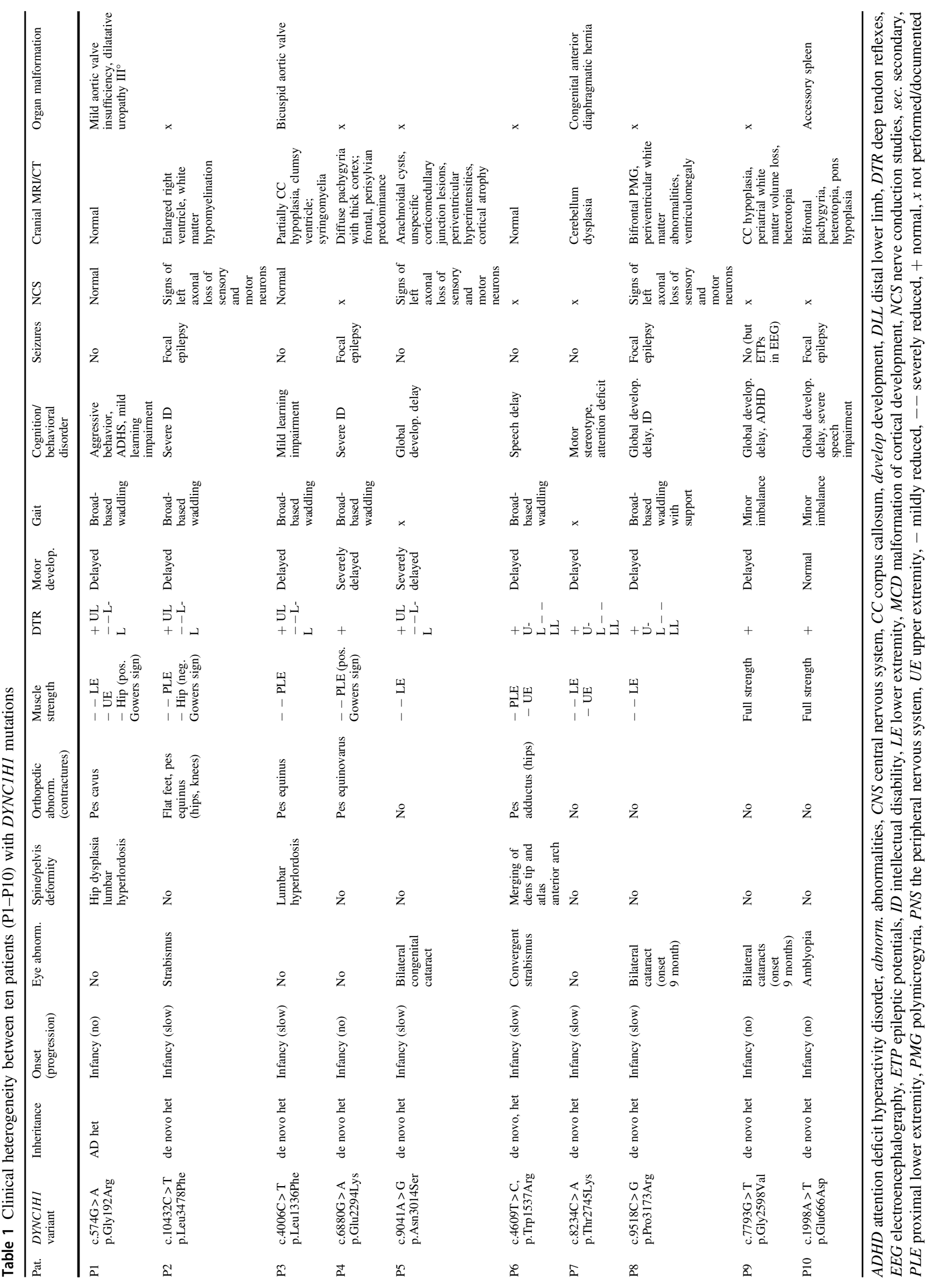


majority having a broad-based waddling gait (P1-P4, P6, and P8) and two with only minor imbalance (P9 and P10). All patients had varying degrees of neurodevelopmental delay, ranging from mild learning impairment, via speech developmental delay (P6, P10) to global developmental delay (P5, P10) or mild (P3, P8) or severe ID (P4).

Information about nerve conduction velocities were available in five patients, with three patients having signs of axonal impairment of sensory and motor neurons (P2, P5, and P7).

Early onset epilepsy was diagnosed in $\mathrm{P} 2, \mathrm{P} 4, \mathrm{P} 8$, and P10 at a median age of 27 months (range 11-48 months). Most patients remained seizure free with single or combined anticonvulsive medication, except the therapy-refractory course of $\mathrm{P} 4$, where levetiracetam and ketogenic diet merely reduced seizure frequency (Table 1). Magnetic resonance imaging (MRI) revealed brain anomalies in all patients except P1 and P6, with ventricle abnormalities (P2, P3, and $\mathrm{P} 7)$, pachygyria (P4 and $\mathrm{P} 10)$, corpus callosum hypoplasia (P3 and P9), pons hypoplasia (P10), and cerebellar dysplasia (P7) (Table 1, Fig. 3).

Furthermore, we identified extra nervous system symptoms, irrespective of the predominant clinical presentation, i.e., (i) facial dysmorphism, macrocephaly (P2), (ii) ophthalmologic anomalies (strabism, (P2, P6), amblyopia (P10), congenital cataracts (P5, P8, and P9)), (iii) osteocutaneous anomalies (e.g., prominent calcanei, cutis laxa, P5), (iv) syringomyelia (P3), (v) bicuspid aortic valve without stenosis but mild insufficiency, (vi) mild aortic valve insufficiency (P1), (vii) congenital diaphragmatic hernia (P7), (viii) dilatative uropathy (P1), and (ix) an accessory spleen (P10) (Table 1). The father of P1, who carries the same variant, has no definite signs or symptoms of a NMD, but he has never been athletic and has developed bilateral hip anthropathy around the age of 40 years. A simular case of a family member with the same mutation as a severely affected individual has been described in the literature before [37].

\section{Domain-specific review of genotype-phenotype correlations in the literature}

As described in the methods, we performed a review of the

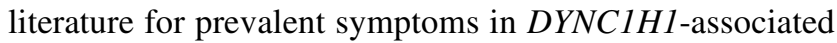
disorders and portrayed the results in a balloon plot using $\mathrm{R}$ (Fig. 4b, c). The genotype-phenotype analyses on our patients and the patients found in the literature revealed a significant difference between the different domains of DYNCIH1. The statistical analyses revealed that specific mutations in the dimerization domain of $D Y N C 1 H 1$ corresponded to a NMD phenotype in reported patients with reductions in lower limb strength and mostly preserved upper limb strength (DYNC1H1-NMD, Fig. 4b). For the detailed results of the statistical tests, please see Supplementary Table 3.
For patients with specific MRI findings and symptoms associated with NDD, e.g., ID, behavioral abnormalities, and seizures, we perceived that the patients reported in the literature were largely spared from mutations in the dimerization domain (Fig. 4c). Instead, seizures were mostly reported in the motor domain, and ID and behavioral abnormalities were largely reported in the beginning tail, linker, and motor domains. MRI abnormalities were largely reported in the motor domain, specifically pachygyria.

\section{Genomic analysis and profiling}

We identified nine heterozygous novel pathogenic or likely pathogenic variants in $D Y N C 1 H 1$; P9's variant was reported previously in ClinVar (https://www.ncbi.nlm.nih.gov/clinvar/) (Table 1, Fig. 1a-e). All variants described were missing in population databases, classified as likely pathogenic according to the ACMG criteria and were evaluated based on gene constraint statistics (Fig. 1, Table 2). Three mutations were in the tail domains ( $\mathrm{P} 1, \mathrm{P} 3$, and $\mathrm{P} 10)$, one mutation was in the linker domains (P6), four mutations were in the motor domains ( $\mathrm{P} 4, \mathrm{P} 5, \mathrm{P} 7$, and $\mathrm{P} 9)$, and two mutations in the stalk or MTBD (P2 and P8) (Fig. 1b). Two mutations were located inside $(\mathrm{P} 1, \mathrm{P} 2)$ and three mutations close to the coiled coil (CC) domains (P3, P6, and P8), four mutations in the AAA domains (AAA2: P4; AAA3: P7, P9; AAA4: P5) and one in a DYNC1LI2 interaction domain (P10).

We performed additional statistical analyses for variant pathogenicity with multiple prediction tools as described in the methods, which projected the variants in our patient collective to have a highly damaging impact (Fig. 1c-e, Table 2, Supplementary Table 2).

In the comparison between subjects with a two-factor ANOVA, we observed significant differences in CADDPhred values between the reports "healthy subjects" (mean: 23.809; standard error of mean: 0.25) and "patients" (mean: 29.05; standard error of mean: 0.612) $(p<0.001)$. When differentiating the patient groups in a post hoc test (Bonferroni), there were significant differences between the groups "gnomAD variants" and "ClinVar variants (likely pathogenic and pathogenic)" (two-factor ANOVA, $p<0.01$ ), and "gnomAD variants" and "patients' variants" from our collective (two-factor ANOVA, $p<0.05$, Fig. 1d, e).

In-depth interaction analyses for CADD-Phred score values uncovered a significant interaction between the group "reports" (patients vs healthy) and "regions" (above-mentioned protein domains) (two-factor ANOVA, $p<0.05$ ). Specifically, in the linker domain, the mutations from patients showed significantly higher mean CADD-Phred score values than the variants in healthy subjects (scores in healthy group 23.1 vs patients 32.3, two-factor ANOVA, $p<0.001$ ), while other domains showed less drastic differences in the 
Fig. 3 Brain abnormalities found in patients with mutations in DYNC1H1. The arrow in the figure points out the brain abnormality described in the figure legend. a Sagittal and b axial $\mathrm{T} 1$ weighted image of P6 at the age of 3 years showing normal brain anatomy. c Axial FLAIR sequences in P5 at 23 months-of-age showing periventricular lesions and cortical atrophy. d Sagittal T1 weighted MRI of P9 at the age of 7 years, revealed thinning of posterior body of corpus callosum. e Axial T2-weighted MRI of P9, revealed enlarged posterior horns of lateral ventricles and periatrial white matter volume loss. f Axial T2 weighted MRI of P9, indicating cortical heterotopia in left fronta deep white matter. g Sagittal, $\mathbf{h}$ coronal, and $\mathbf{i}$ axial T2weighted MRI of P4 at the age of 7 months-of-age showing diffuse pachygyria with cortical thickness $>10 \mathrm{~mm}$ with frontal and perisylvian predominance. j Sagittal and k transversal T2weighted MRI of P7 at the age of 4 years showing an isolated dysplasia of the right cerebellum and cerebellar vermis with an enlargement of the fourth ventricle on the left and notably without the involvement of the pons or mesencephalon.

I Transversal T2-weighted MRI of P5 at 23 months-of-age showing in contrast to $\mathbf{k}$ no vermis or ventricle enlargement. m Sagittal T2-weighted MRI of P10 at the age of 4 years showing brain stem hypoplasia. n Axial T2-weighted MRI of P10 showing bilateral temporal and parietal pachygyria reaching until dorsal part of the frontal lobe. o Axial T2-weighted MRI of P5 at 23 months-of-age showing cortical brain atrophy without pahygyria

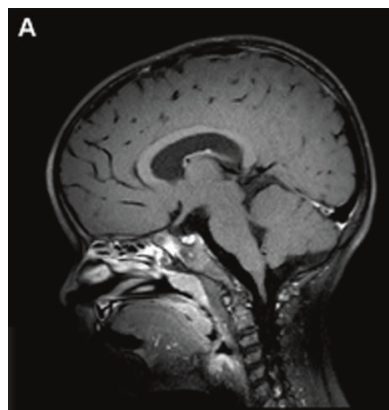

D
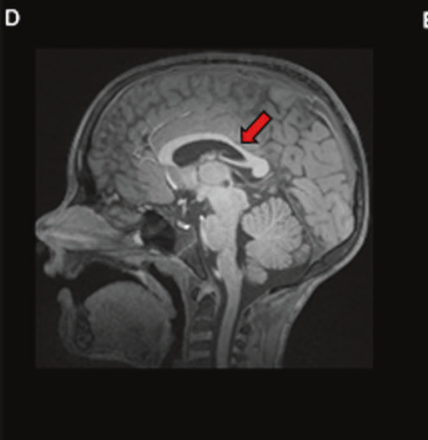

G
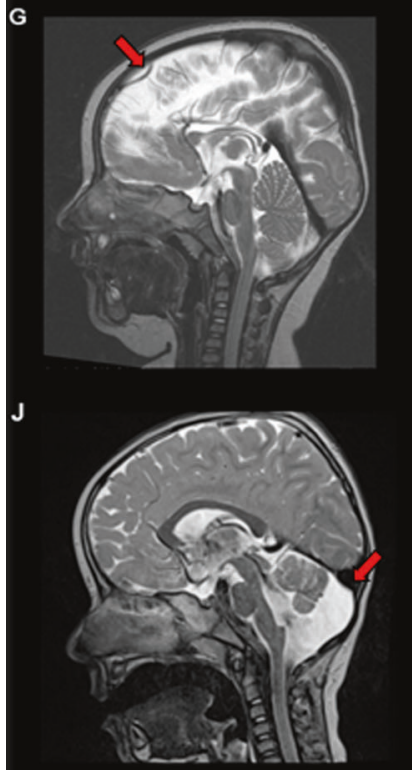

M

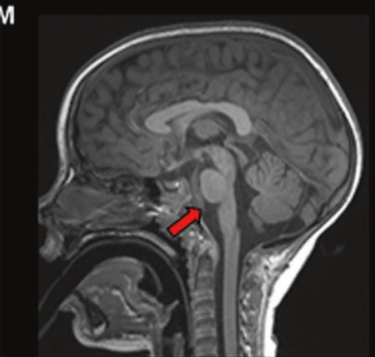

B

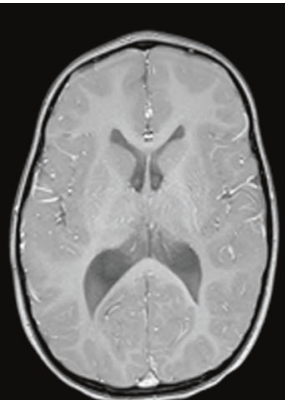

C
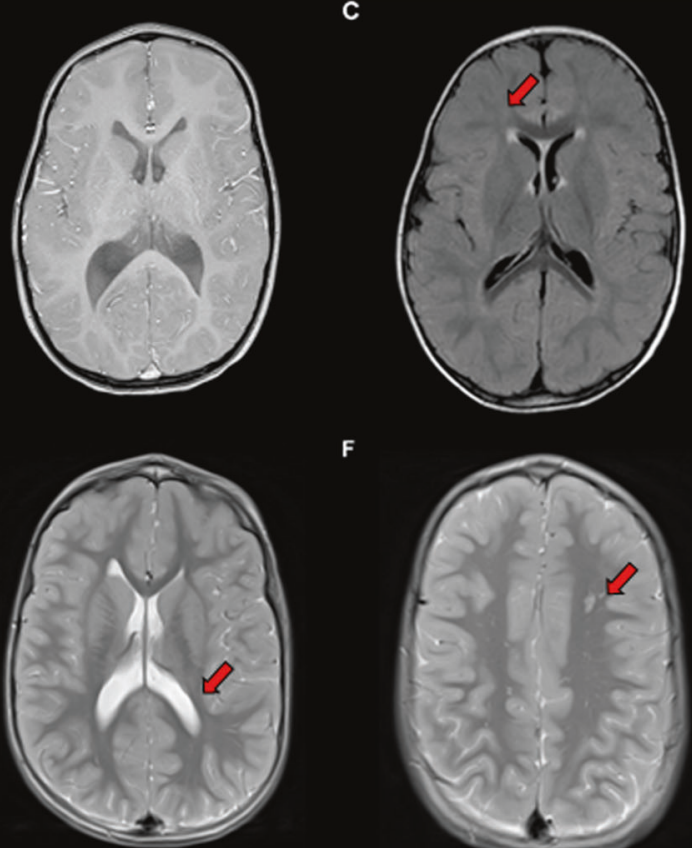

H
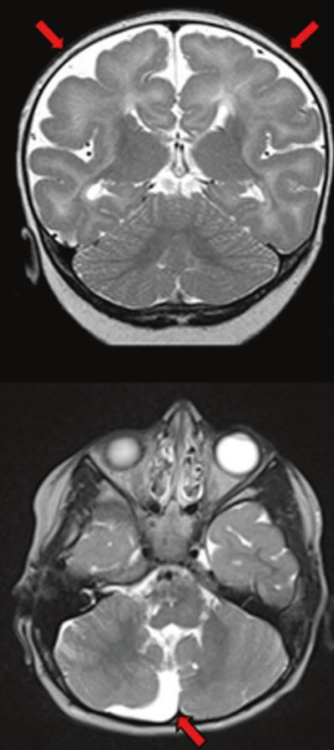

N

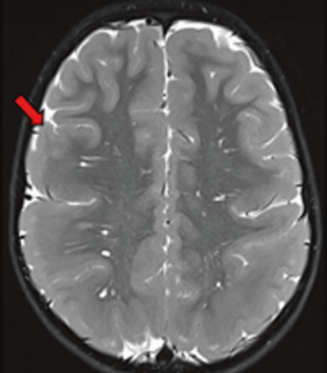

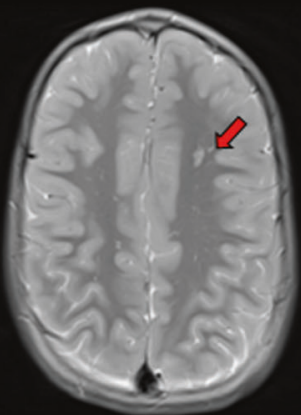
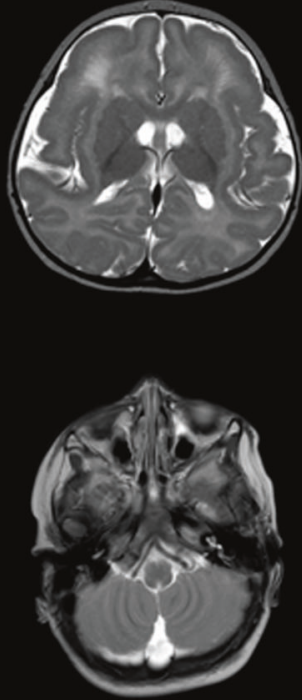

○

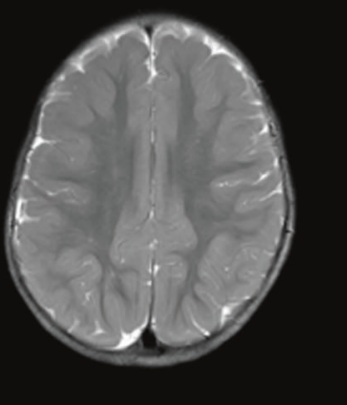




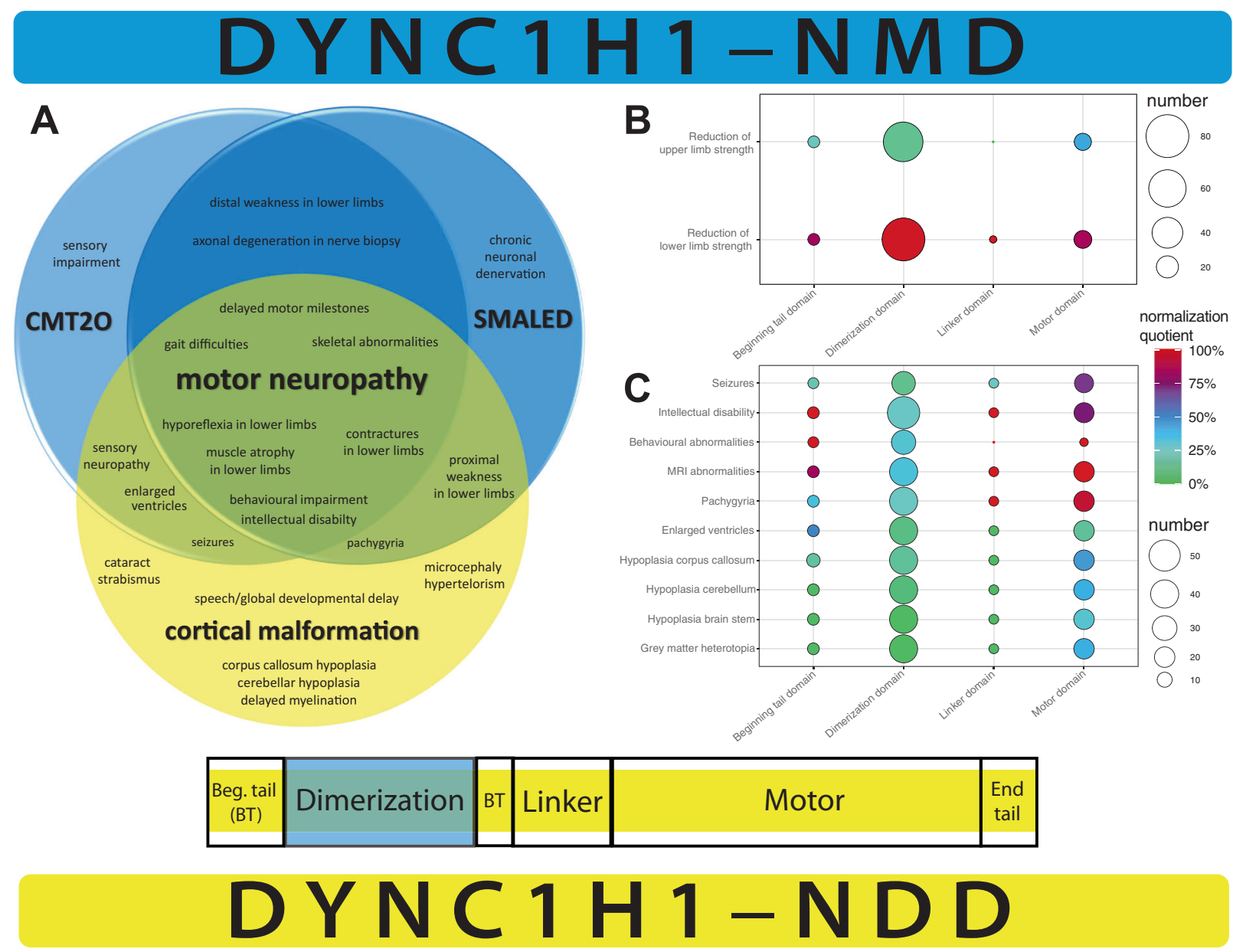

Fig. 4 Overview of overlapping clinical disease manifestation of DYNC1H1-associated disorders and domain-specific presentation of genotype-phenotype correlation based on literature review. a On the left, Venn diagram of the recorded symptoms in patients with each of the three known entities associated with $\mathrm{DYNC1H1}$ mutations and the overlap of phenotypes in DYNC1H1-associated disorders: CharcotMarie-Tooth disease Type 20 (CMT20), lower extremity-predominant spinal muscular atrophy (SMALED), and cortical malformations. The symptoms were taken from an extensive PubMed literature search ("dync1h1", with each "motor neuropathy", "CMT20", "charcotmarie-tooth", "SMALED", "spinal muscular atrophy", "malformation", "MRD13", "mental retardation"). Specifically, neuromuscular symptoms as in CMT20 and SMALED were mostly observed in patients with mutations in the dimerization domain and cortical malformation was mostly observed in motor domain mutations. On the right, a simplified overview of the protein model from Fig. $1 \mathrm{~b}$. b Balloon plot for symptoms "reduction of upper limb strength" and "reduction of lower limb strength" recorded in the literature search with mutations in the beginning tail, dimerization, linker, and motor domains. The size of patient groups denoted with the size of circles on the right (smallest circle 20, biggest circle 80). The calculated

CADD-Phred values between healthy variants and patient mutations (tail 23.6 vs 27.9 , motor 24.8 vs 28.5 , MTBD 23.7 vs 27.6, two-factor ANOVA, all $p<0.001$ ).

In the next step, we evaluated the intolerance toward missense mutations throughout the regions of $D Y N C 1 H 1$. normalization quotient (from green to blue to red, on the right) from Pearson's chi-square test as described in methods revealed clustering of reductions of lower limb strength with preserved upper limb strength in the dimerization domain. c Balloon plot for symptoms "seizures", "intellectual disability", "behavioral abnormalities", "MRI abnormalities" in general, "pachygyria", "enlarged ventricles", "hypoplasia corpus callosum", "hypoplasia cerebellum", "hypoplasia brain stem", and "gray matter heterotopia" recorded in the literature search with mutations in the beginning tail, dimerization, linker, and motor domains. The size of patient groups denoted with the size of circles on the right (smallest circle 10, biggest circle 50). The calculated normalization quotient from Pearson's chi-square test as described in methods revealed clustering of intellectual disability and behavioral abnormalities in patients with mutations in the beginning tail, linker, and motor domains. Seizures and all MRI abnormalities specifically clustered in patients with mutations in the motor domain. Patients with mutations in the dimerization domain were largely spared of these symptoms, thus underlining the hypothesis that DYNC1H1-NMD and -NDD can be traced to specific domain mutations

The MTR for our patients' variants showed that these variants were in regions of high intolerance toward missense variations (Fig. 1c). In the comparison between subjects with a two-factor ANOVA, we tested for differences in MTR score values between healthy subjects and patients 


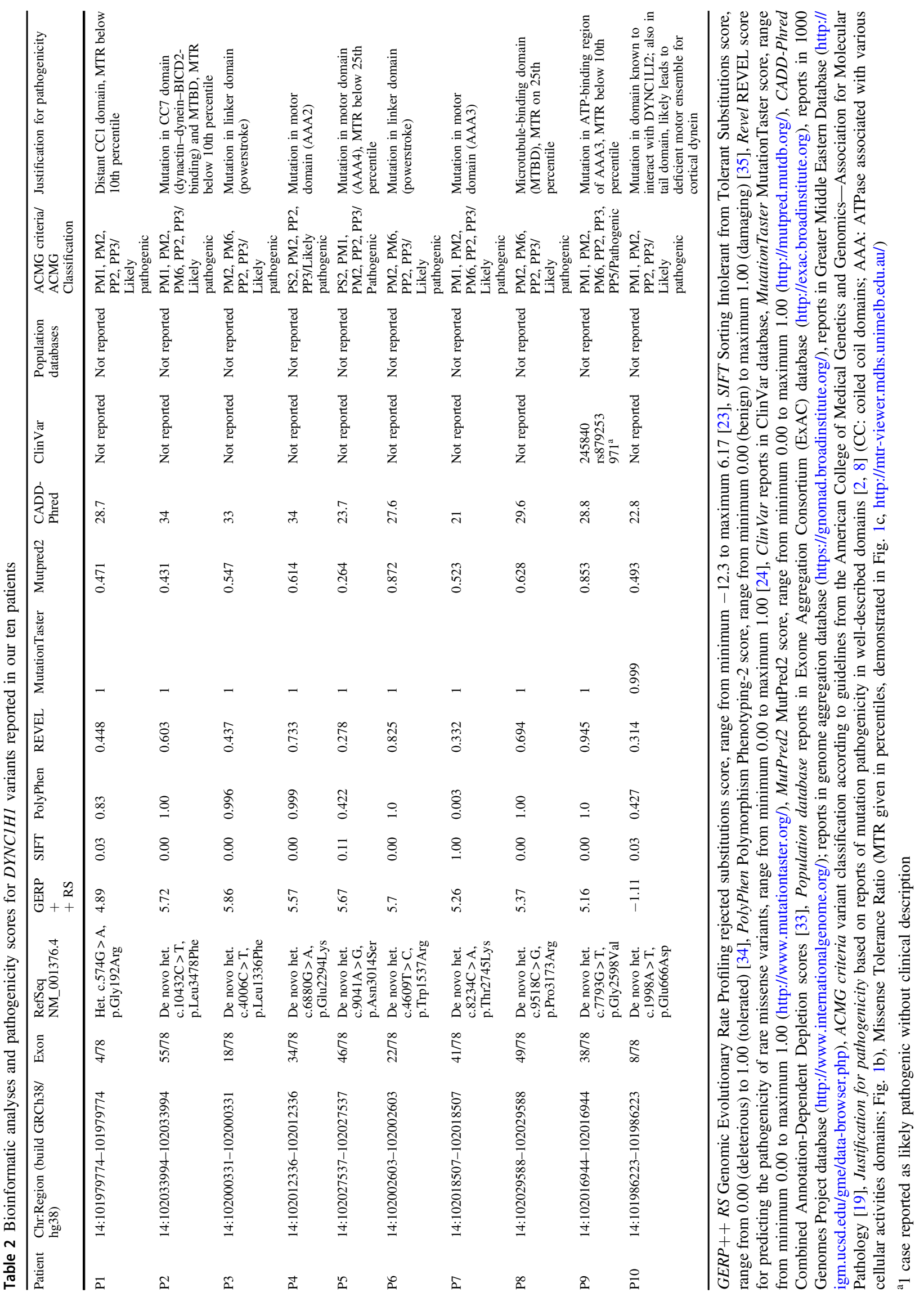


and observed a significantly higher MTR score values of 0.63 for variants in the healthy population $(0.63)$ than in the patients mutations $(0.46)$, i.e., patient mutations were at amino acid residues with higher intolerance toward missense mutations $(p<0.001)$.

In-depth interaction analyses for MTR score values also revealed significant differences in MTR scores between protein regions (two-factor ANOVA, $p<0.001$ ), while mean MTR scores decreased over protein length (tail $0.63>$ linker $0.55>$ motor $0.55>$ MTBD 0.48 ). In a post hoc test (Bonferroni), we tested for the interactions between reports (healthy vs patients) and protein regions and observed that variants in the linker domain had the highest mean MTR scores in the healthy population (0.72), but the lowest mean MTR scores in patients (0.39) (two-factor ANOVA, $p<0.001$ ). Mean MTR scores for other domains also showed dissociations between healthy subjects and patients (tail 0.68 vs 0.57 , motor 0.65 vs 0.45 , two-factor ANOVA, all $p<0.001$ ), while the calculation for MTBD showed similar values between the groups $(0.46$ vs 0.44, two-factor ANOVA, $p<0.001$ ).

\section{Discussion}

We report ten patients with nine novel mutations in the DYNC1H1 gene (Table 2). In neurons, DYNC1H1 as part of the cytoplasmic dynein complex is essential for retrograde transport of cargos in axons and dendrites, thus involved in neuronal development, morphology, and survival [7, 9, 45].

In the literature, an increasing number of phenotype expansions have shown an overlapping phenotype link between motor neuropathies and brain malformations $[32,37,46,47]$. We propose a novel clinical classification of $\mathrm{DYNC} 1 \mathrm{H1}$-related disease entities that follows a holistic approach, focusing on the patients' individual but complex clinical traits in the center of the classification, rather than the current reductionistic classification (e.g., SMALED or MRD13): $D Y N C 1 H 1$-related disorders with an exclusive NMD phenotype, DYNC1H1-NMD, and a combined NMD-CNS phenotype, $D Y N C 1 H 1-\mathrm{NDD}$, on either sides of the spectrum (Fig. 4).

\section{Genomic profiling of population datasets for the domain-specific impact of genetic variation}

All mutations in our ten patients showed high CADD scores, high intolerance toward missense variations (MTR, Fig. 1c) in evolutionary well-conserved domains (Fig. 2), and no reports in population databases (Table 2). When comparing healthy with patient report datasets, we observed that pathogenic mutations with significantly higher CADD scores in patients (Table 2) in all domains due to evolutionary conservation and drastic effects on physicochemical properties cause by amino acid exchanges at the mutational sites $(p<0.001)$. This is also evident from multiple lines of in silico pathogenicity scores (Supplementary Table 2). Next, we looked at domain-specific statistical pathogenicity prediction tools. The significant interaction between reports and protein regions revealed particularly higher CADDPhred score value increases in the linker domain (healthy 23.1 vs patients $32.2, p<0.001$ ), which hints at an evolutionary well-conserved domain (Fig. 2).

The tail domain itself shows rather low conservation throughout species and variants in the tail domains can be observed quite often in human, thus CADD-Phred score values in the healthy dataset are rather low. The tail domain mutations in patients tend to have rather high CADD-Phred score values and are situated at residues with rather high tolerance toward missense variants. The $\mathrm{CC}$ regions in the tail domains are highly conserved are also connoted with high CADD-Phred score values in patient mutations. As the CADD scores also comprise protein impact tools (e.g., Grantham, SIFT, and PolyPhen), the amino acid exchanges in the patients mutations show higher CADD scores and more drastic effects in physicochemical properties than in the healthy dataset. Clustered around the $\mathrm{CC}$ regions, the MTR scores show significantly higher intolerance toward missense mutations $(p<0.001)$.

The CADD and MTR score values further support that the motor domain is highly conserved and the mutational effects lead to drastic changes on the protein level. MTBD domain variants in the healthy and patient datasets show rather similar MTR scores, thus a similarly high intolerance toward missense variants. When comparing Fig. 1c, d, we note that there is a much lower number of variants as well as significantly lower mean CADD score value in the MTBD $(p<0.001)$, i.e., MTBD variants are generally scarce in healthy population datasets and the protein level scores do not show drastic effects in physiochemical properties.

Based on this genomic profiling of healthy and patient variant datasets, we underline the domain-specific effects of genetic variation in $\mathrm{DYNC} 1 \mathrm{H1}$ and we further recommend to interpret $\mathrm{DYNC} 1 \mathrm{H1}$ variants based on the following model:

(1) Region location/ conservation: mutations in the linker, motor, and MTBD region are in highly evolutionarily conserved domains with important functional roles in processive and powerstroke movements.

(2) Gene-wide missense intolerance: as a measure of regional intolerance to missense variation, the spatial distribution of observed vs expected variants have to be evaluated concerning the healthy population. Patient mutations are situated at genomic coordinates with significantly lower MTR scores, i.e., are more prone to intolerance from missense variation. 
(3) Protein level change in physicochemical property: based on multiple lines of pathogenicity prediction scoring tools, the effect of the amino acid exchange in a mutation can be evaluated for missense variations for protein structural and functional properties, including secondary structure, solvent accessibility, functional domains, methylation, phosphorylation, and glycosylation.

\section{DYNC1H1-NMD}

In our cohort, no patient was characterized by exclusive DYNC1H1-NMD. In the literature, about half of patients reported an exclusive NMD phenotype, predominantly involving the lower limbs (SMALED, CMT20) [1, 30] and presenting with delay in motor milestones, muscle weakness, atrophy hyporeflexia, and skeletal limb abnormalities. Most affected individuals developed secondary orthopedic symptoms like hyperlordosis and feet deformities [32]. However, patients did not exhibit CNS involvement, e.g., ID or cortical malformations (Fig. 4). DYNClH1 mutations in patients with NMD were located in the tail domain of DYNCIHI (53AA-1867AA), predominantly within the dimerization domain (300AA-1140AA) (Fig. 1a-d). Previous studies demonstrated that these tail domain mutations show no disruption in the retrograde movement of dynein along microtubules, in contrast to motor domain mutations. They rather exclusively shortened the run length of processive dynein-dynactin-BICD2N complexes, leading to a possible disruption of neuronal cargo delivery [8]. A hypothesis why the muscular atrophy in DYNC1H1-NMD affects predominantly the lower extremities is that neuronal transportation distance is longer compared with the upper extremity or the cortex, thus being more sensitive by a deduction in run length [8]. Further studies need to evaluate if extrinsic (environmental) or intrinsic factors (methylation, genome interactions) contribute to phenotypic variability.

\section{DYNC1H1-NDD}

A second group of patients presented DYNCIH1-NMD with concomitant CNS involvement. In our cohort, all patients presented with predominant lower extremity muscle atrophy, a variable degree of ID, global developmental delay, and/or brain malformations in MRI.

From a recent molecular biological study, mutations in the linker region were observed with deficient powerstroke movements [2]. CC mutations (P1) displayed altered foci for plus-end dynein (dynactin independent) and cortical dynein (dynactin dependent). Furthermore, these mutations resulted in processive movement reductions of the dynein complex [8]. Mutations in MTBD are known to be associated with a reduction in velocity, displacement, and neck transit success, all of which are essential for the stabilization and advancement of movements [8] leading to a more severe disruption of motor activity like in the other variants associated with $D Y N C 1 H 1-N D D$. The fact that MTBD domain mutations do not appear in healthy datasets hints at the possibility of early onset lethal disease courses for MTBD domain mutations. Motor domain mutations, with the six AAA domains forming the core complex, were associated with microtubule gliding defects (AAA5) or inhibition of any movement (AAA1), whereas other protein areas (tail, linker, or MTBD) do not alter glinding [8]. Moreover, mutations lead to static binding to microtubule (AAA1) or diffuse binding behavior (AAA5 and MTBD), both resulting in disturbed motor activity and possibly secondarily in severe disruption of neuronal migration and myelination [8, 45]. We identified four AAA domain mutations with NMD, cognitive-behavioral impairment, and brain malformation (P4, P5, P7, P9, Fig. 1a-d). These findings highlight that motor domain mutations lead to MCD due to a more severe disruption of the dynein movement.

P2 displayed cerebral hypomyelination on brain MRI with a mutation in $\mathrm{CC} 7$, which is involved in the sedimentation of microtubules with the MTBD in the context of a fusion protein with a heterologous CC [8].

Patients with $D Y N C 1 H 1-N D D$ had different degrees of ID, learning, and language impairment, in line with our findings. Some reports described more severely affected individuals with epilepsy or spastic paraplegia and variable MCD including pachygyria and polymicrogyria, frequently in combination with ventricular anomalies, abnormal white matter and corpus callosum, and cerebellar hypoplasia [5, 37]. Almost all of our patients had an overlapping phenotype with PNS and CNS involvement, signifying a clinical continuum (Fig. 4). Of note, all patients exhibited rare signs and symptoms closely linked to neuronal development (e.g., cataracts, syrinx, etc.), even patients with tail domain mutations (Table 1).

We propose a novel clinical classification of $\mathrm{DYNClHI}$ related disease entities that follows a holistic approach, focusing on the patients' individual but complex clinical traits in the center of the classification, rather than the current reductionistic classification (e.g., SMALED or MRD13). Our

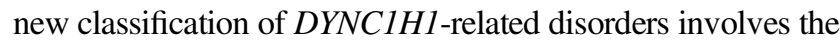
leading phenotype characteristics, i.e., $D Y N C 1 H 1-\mathrm{NMD}$ as a NMD phenotype and DYNC1H1-NDD with concomitant CNS involvement.

\section{Data availability}

Any data not published within the article is available as anonymized data and will be shared by request from the 
authors. The next-generation sequencing raw data including either gene panel or WES cannot be fully shared publicly since this may facilitate the de-anonymization of the study subjects and also infringe their privacy. We also do not have ethical permission to share the full and raw next-generation sequencing data or full variant list from it individually.

Acknowledgements We would like to thank the patients and their families of this multi-center study. We furthermore thank Özkan Özdemir, PhD for support in the bioinformatics analyses, Dr.med. Annegret Holm for clinical information, and the Regional Computing Center of the University of Cologne (RRZK) for providing computing time for the bioinformatics analyses on the DFG-funded HighPerformance Computing (HPC) system CHEOPS as well as support. This work was supported by the German Research Foundation (DFG; Emmy Noether Grant CI 218/1-1 to SC and FO3004, SFB1315 to AMK), the Cologne Clinician Scientist Program (CCSP) to HSD via DFG FI 773/15-1, and Gerok Program/Faculty of Medicine/University of Cologne (to HSD), and the Charité-Universitätsmedizin Berlin (to AMK). The MYO-SEQ project was funded by Sanofi Genzyme, Ultragenyx, LGMD2I Research Fund, Samantha J Brazzo Foundation, LGMD2D Foundation, Kurt+Peter Foundation, Muscular Dystrophy UK, and Coalition to Cure Calpain 3.

Funding Open access funding provided by Projekt DEAL.

Author contributions LLB and HSD analyzed clinical patient data, neuroimaging, molecular genetics, and bioinformatic data, and wrote the manuscript. JS, DA, MS, FP, TS, LB, LR, SS, BW, KJ, VS, AMK, IK, SC, and NDD analyzed clinical patient and genetic data and critically reviewed the manuscript. SC and MVDH designed and supervised the study, obtained funding, and reviewed the manuscript. All authors critically reviewed and approved the final manuscript and agree to be accounted for all aspects of the work.

Data sharing Anonymized information of all participants was made available and shared only among qualified investigators.

\section{Compliance with ethical standards}

Conflict of interest VS reports grants from Sanofi Genzyme Ultragenyx, Muscular Dystrophy UK, The Samantha J. Brazzo Foundation, LGMD2iFund, LGMD2D Foundation, Coalition to Cure Calpain 3, Kurt + Peter Foundation, during the conduct of the study. The other authors declare no conflict of interest.

Publisher's note Springer Nature remains neutral with regard to jurisdictional claims in published maps and institutional affiliations.

Open Access This article is licensed under a Creative Commons Attribution 4.0 International License, which permits use, sharing, adaptation, distribution and reproduction in any medium or format, as long as you give appropriate credit to the original author(s) and the source, provide a link to the Creative Commons license, and indicate if changes were made. The images or other third party material in this article are included in the article's Creative Commons license, unless indicated otherwise in a credit line to the material. If material is not included in the article's Creative Commons license and your intended use is not permitted by statutory regulation or exceeds the permitted use, you will need to obtain permission directly from the copyright holder. To view a copy of this license, visit http://creativecommons. org/licenses/by/4.0/.

\section{References}

1. Harms MB, Allred P, Gardner R Jr, Fernandes Filho JA, Florence J, Pestronk A, et al. Dominant spinal muscular atrophy with lower extremity predominance: linkage to $14 q 32$. Neurology. 2010;75:539-46.

2. Marzo MG, Griswold JM, Ruff KM, Buchmeier RE, Fees CP, Markus SM. Molecular basis for dyneinopathies reveals insight into dynein regulation and dysfunction. Elife. 2019;8:e47246.

3. Strickland AV, Schabhuttl M, Offenbacher H, Synofzik M, Hauser NS, Brunner-Krainz M, et al. Mutation screen reveals novel variants and expands the phenotypes associated with DYNC1H1. J Neurol. 2015;262:2124-34.

4. Weedon MN, Hastings R, Caswell R, Xie W, Paszkiewicz K, Antoniadi $\mathrm{T}$, et al. Exome sequencing identifies a DYNC1H1 mutation in a large pedigree with dominant axonal Charcot-MarieTooth disease. Am J Hum Genet. 2011;89:308-12.

5. Willemsen MH, Vissers LE, Willemsen MA, van Bon BW, Kroes T, de Ligt J, et al. Mutations in DYNC1H1 cause severe intellectual disability with neuronal migration defects. J Med Genet. 2012;49:179-83.

6. Reck-Peterson SL, Redwine WB, Vale RD, Carter AP. The cytoplasmic dynein transport machinery and its many cargoes. Nat Rev Mol Cell Biol. 2018;19:382-98.

7. Hirokawa N, Niwa S, Tanaka Y. Molecular motors in neurons: transport mechanisms and roles in brain function, development, and disease. Neuron. 2010;68:610-38.

8. Hoang HT, Schlager MA, Carter AP, Bullock SL. DYNC1H1 mutations associated with neurological diseases compromise processivity of dynein-dynactin-cargo adaptor complexes. Proc Natl Acad Sci USA. 2017;114:E1597-606.

9. Schiavo G, Greensmith L, Hafezparast M, Fisher EM. Cytoplasmic dynein heavy chain: the servant of many masters. Trends Neurosci. 2013;36:641-51.

10. Cianfrocco MA, DeSantis ME, Leschziner AE, Reck-Peterson SL. Mechanism and regulation of cytoplasmic dynein. Annu Rev Cell Dev Biol. 2015;31:83-108.

11. Johnson K, Topf A, Bertoli M, Phillips L, Claeys KG, Stojanovic $\mathrm{VR}$, et al. Identification of GAA variants through whole exome sequencing targeted to a cohort of 606 patients with unexplained limb-girdle muscle weakness. Orphanet J Rare Dis. 2017;12:173.

12. Di Donato N, Timms AE, Aldinger KA, Mirzaa GM, Bennett JT, Collins S, et al. Analysis of 17 genes detects mutations in $81 \%$ of 811 patients with lissencephaly. Genet Med. 2018;20:1354-64.

13. Di Donato N, Neuhann T, Kahlert AK, Klink B, Hackmann K, Neuhann I, et al. Mutations in EXOSC2 are associated with a novel syndrome characterised by retinitis pigmentosa, progressive hearing loss, premature ageing, short stature, mild intellectual disability and distinctive gestalt. J Med Genet. 2016;53:419-25.

14. Dafsari HS, Sprute R, Wunderlich G, Daimaguler HS, Karaca E, Contreras A, et al. Novel mutations in KMT2B offer pathophysiological insights into childhood-onset progressive dystonia. J Hum Genet. 2019;64:803-13.

15. Dafsari HS, Kawalia A, Sprute R, Karakaya M, Malenica A, Herkenrath $\mathrm{P}$, et al. Novel mutations in SLC6A5 with benign course in hyperekplexia. Cold Spring Harb Mol Case Stud. 2019; 5:a004465.

16. Kawalia A, Motameny S, Wonczak S, Thiele H, Nieroda L, Jabbari $\mathrm{K}$, et al. Leveraging the power of high performance computing for next generation sequencing data analysis: tricks and twists from a high throughput exome workflow. PLoS ONE. 2015; 10:e126321.

17. Ramu A, Noordam MJ, Schwartz RS, Wuster A, Hurles ME, Cartwright RA, et al. DeNovoGear: de novo indel and point mutation discovery and phasing. Nat Methods. 2013;10:985-7. 
18. Neveling K, Feenstra I, Gilissen C, Hoefsloot LH, Kamsteeg EJ, Mensenkamp AR, et al. A post-hoc comparison of the utility of sanger sequencing and exome sequencing for the diagnosis of heterogeneous diseases. Hum Mutat. 2013;34:1721-6.

19. Richards S, Aziz N, Bale S, Bick D, Das S, Gastier-Foster J, et al. Standards and guidelines for the interpretation of sequence variants: a joint consensus recommendation of the American College of Medical Genetics and Genomics and the Association for Molecular Pathology. Genet Med. 2015;17:405-24.

20. Beecroft SJ, McLean CA, Delatycki MB, Koshy K, Yiu E, Hali$\log l u \mathrm{G}$, et al. Expanding the phenotypic spectrum associated with mutations of DYNC1H1. Neuromuscul Disord. 2017;27:607-15.

21. Niu Q, Wang X, Shi M, Jin Q. A novel DYNC1H1 mutation causing spinal muscular atrophy with lower extremity predominance. Neurol Genet. 2015;1:e20.

22. Ding D, Chen Z, Li K, Long Z, Ye W, Tang Z, et al. Identification of a de novo DYNC1H1 mutation via WES according to published guidelines. Sci Rep. 2016;6:20423.

23. Lin Z, Liu Z, Li X, Li F, Hu Y, Chen B, et al. Whole-exome sequencing identifies a novel de novo mutation in DYNC1H1 in epileptic encephalopathies. Sci Rep. 2017;7:258.

24. Chan SHS, van Alfen N, Thuestad IJ, Ip J, Chan AO, Mak C, et al. A recurrent de novo DYNC1H1 tail domain mutation causes spinal muscular atrophy with lower extremity predominance, learning difficulties and mild brain abnormality. Neuromuscul Disord. 2018;28:750-56.

25. Gelineau-Morel R, Lukacs M, Weaver KN, Hufnagel RB, Gilbert DL, Stottmann RW. Congenital cataracts and gut dysmotility in a DYNC1H1 dyneinopathy patient. Genes (Basel). 2016;7:85.

26. Chen J, Yoon SH, Grynpas MD, Mitchell J. Pre-treatment with pamidronate improves bone mechanical properties in Mdx mice treated with glucocorticoids. Calcif Tissue Int. 2019;104:182-92.

27. Laquerriere A, Maillard C, Cavallin M, Chapon F, Marguet F, Molin A, et al. Neuropathological hallmarks of brain malformations in extreme phenotypes related to DYNC1H1 mutations. J Neuropathol Exp Neurol. 2017;76:195-205.

28. Hertecant J, Komara M, Nagi A, Suleiman J, Al-Gazali L, Ali BR. A novel de novo mutation in DYNC1H1 gene underlying malformation of cortical development and cataract. Meta Gene. 2016;9:124-7.

29. Peeters K, Bervoets S, Chamova T, Litvinenko I, De Vriendt E, Bichev S, et al. Novel mutations in the DYNC1H1 tail domain refine the genetic and clinical spectrum of dyneinopathies. Hum Mutat. 2015;36:287-91.

30. Tsurusaki Y, Saitoh S, Tomizawa K, Sudo A, Asahina N, Shiraishi H, et al. A DYNC1H1 mutation causes a dominant spinal muscular atrophy with lower extremity predominance. Neurogenetics. 2012;13: 327-32.

31. Das J, Lilleker JB, Jabbal K, Ealing J. A missense mutation in DYNC1H1 gene causing spinal muscular atrophy-lower extremity, dominant. Neurol Neurochir Pol. 2018;52:293-97.

32. Punetha J, Monges S, Franchi ME, Hoffman EP, Cirak S, Tesi-Rocha C. Exome sequencing identifies DYNC1H1 variant associated with vertebral abnormality and spinal muscular atrophy with lower extremity predominance. Pediatr Neurol. 2015;52:239-44.
33. Harms MB, Ori-McKenney KM, Scoto M, Tuck EP, Bell S, Ma $\mathrm{D}$, et al. Mutations in the tail domain of DYNC1H1 cause dominant spinal muscular atrophy. Neurology. 2012;78:1714-20.

34. Poirier K, Lebrun N, Broix L, Tian G, Saillour Y, Boscheron C, et al. Mutations in TUBG1, DYNC1H1, KIF5C and KIF2A cause malformations of cortical development and microcephaly. Nat Genet. 2013;45:639-47.

35. Zillhardt JL, Poirier K, Broix L, Lebrun N, Elmorjani A, Martinovic $\mathrm{J}$, et al. Mosaic parental germline mutations causing recurrent forms of malformations of cortical development. Eur $\mathrm{J}$ Hum Genet. 2016;24:611-4.

36. Fiorillo C, Moro F, Yi J, Weil S, Brisca G, Astrea G, et al. Novel dynein DYNC1H1 neck and motor domain mutations link distal spinal muscular atrophy and abnormal cortical development. Hum Mutat. 2014;35:298-302.

37. Scoto M, Rossor AM, Harms MB, Cirak S, Calissano M, Robb S, et al. Novel mutations expand the clinical spectrum of DYNC1H1associated spinal muscular atrophy. Neurology. 2015;84:668-79.

38. Jamuar SS, Lam AT, Kircher M, D'Gama AM, Wang J, Barry BJ, et al. Somatic mutations in cerebral cortical malformations. N Engl J Med. 2014;371:733-43.

39. Ghosh SG, Becker K, Huang H, Dixon-Salazar T, Chai G, Salpietro $\mathrm{V}$, et al. Biallelic mutations in ADPRHL2, encoding ADPribosylhydrolase 3, lead to a degenerative pediatric stress-induced epileptic ataxia syndrome. Am J Hum Genet. 2018;103:431-9.

40. Abecasis GR, Auton A, Brooks LD, DePristo MA, Durbin RM, Handsaker RE, et al. An integrated map of genetic variation from 1,092 human genomes. Nature. 2012;491:56-65.

41. Scott EM, Halees A, Itan Y, Spencer EG, He Y, Azab MA, et al. Characterization of Greater Middle Eastern genetic variation for enhanced disease gene discovery. Nat Genet. 2016;48:1071-6.

42. Davydov EV, Goode DL, Sirota M, Cooper GM, Sidow A, Batzoglou S. Identifying a high fraction of the human genome to be under selective constraint using GERP ++ . PLoS Comput Biol. 2010;6:e1001025.

43. Ioannidis NM, Rothstein JH, Pejaver V, Middha S, McDonnell SK, Baheti S, et al. REVEL: an ensemble method for predicting the pathogenicity of rare missense variants. Am J Hum Genet. 2016;99:877-85.

44. Li GC, Forster-Benson ETC, Sanders CR. Genetic intolerance analysis as a tool for protein science. Biochim Biophys Acta (BBA)-Biomembr. 2019;1862:183058.

45. Yang ML, Shin J, Kearns CA, Langworthy MM, Snell H, Walker $\mathrm{MB}$, et al. CNS myelination requires cytoplasmic dynein function. Dev Dyn. 2015;244:134-45.

46. Dafsari HS, Byrne S, Lin JP, Pitt M, Jongbloed JD, Flinter F, et al. Goldberg-Shprintzen megacolon syndrome with associated sensory motor axonal neuropathy. Am J Med Genet Part A. 2015; 167:1300-4.

47. Bamborschke D, Pergande M, Becker K, Koerber F, Dotsch J, Vierzig A, et al. A novel mutation in sphingosine-1-phosphate lyase causing congenital brain malformation. Brain Dev. 2018;40:480-83.

48. Rentzsch P, Witten D, Cooper GM, Shendure J, Kircher M. CADD: predicting the deleteriousness of variants throughout the human genome. Nucleic Acids Res. 2019;47:D886-94. 


\section{Affiliations}

\section{Lena-Luise Becker ${ }^{1}{ }^{1} \cdot$ Hormos Salimi Dafsari $^{2} \cdot$ Jens Schallner $^{3} \cdot$ Dalia Abdin $^{4,5} \cdot$ Michael Seifert $^{6}$.} Florence Petit $\mathbb{D}^{7,8} \cdot$ Thomas Smol $\mathbb{D D}^{7,9} \cdot$ Levinus Bok $^{10} \cdot$ Lance Rodan $^{11} \cdot$ Ingrid Krapels $^{12} \cdot$ Stephanie Spranger $^{13}$.

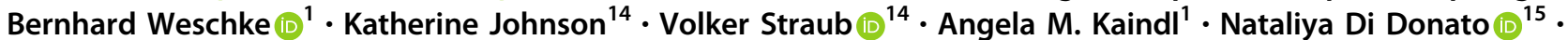

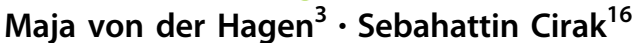

1 Charité-Universitätsmedizin Berlin, Department of Neuropediatrics, Center for Chronically Sick Children, Institute of Cell- and Neurobiology, Berlin, Germany

2 Department of Pediatrics, Faculty of Medicine and University Hospital Cologne, Center for Molecular Medicine Cologne (CMMC), University of Cologne, Cologne, Germany

3 Department of Neuropediatrics, Medizinische Fakultät Carl Gustav Carus, Technische Universität Dresden, Dresden, Germany

4 Carl Gustav Carus Faculty of Medicine, Institute for Clinical Genetics, Technische Universität Dresden, Dresden, Germany

5 Human Cytogenetics Department, National Research Centre, Cairo, Egypt

6 Carl Gustav Carus Faculty of Medicine, Institute for Medical Informatics and Biometry, Dresden, Germany

7 University of Lille, EA 7364-RADEME, Lille, France

8 CHU Lille, Hôpital Jeanne de Flandre, Service de Génétique Clinique, Avenue Eugène Avinée, Lille, France
9 CHU Lille, Hôpital Jeanne de Flandre, Service de Génétique Clinique, Institut de Génétique Médicale, Lille, France

10 Department of Pediatrics, Máxima Medical Center, Veldhoven, The Netherlands

11 Department of Pediatrics and Neurology, Division of Genetics and Genomics, Boston Children's Hospital, Boston, MA, USA

12 Department of Clinical Genetics Maastricht, University Medical Center, Maastricht, The Netherlands

13 Practice for Human Genetics, Bremen, Germany

14 John Walton Muscular Dystrophy Research Centre, Newcastle University and Newcastle Hospital NHS Foundation Trust, Newcastle upon Tyne, UK

15 Carl Gustav Carus Faculty of Medicine, Institute for Clinical Genetics, Technische Universität Dresden, Dresden, Germany

16 Department of Pediatrics, Faculty of Medicine and University Hospital Cologne, Center for Molecular Medicine Cologne (CMMC), Center for Rare Diseases, University of Cologne, Cologne, Germany 